\title{
FINITE SUPERELEMENTS METHOD FOR ELASTICITY PROBLEMS ${ }^{1}$
}

\author{
M. GALANIN ${ }^{1}$, E. SAVENKOV ${ }^{2}$ and J. TEMIS ${ }^{3}$ \\ ${ }^{1,2}$ Keldysh Institute of Applied Mathematics of RAS \\ Russia, 125047, Moscow, Miusskaya Sq., 4 \\ E-mail: galan@keldysh.ru; savenkov@keldysh.ru \\ ${ }^{3}$ Central Institute of Aviation Motors n.a. P.I. Baranov \\ Russia, 111116, Moscow, Aviamotornaya st., 2 \\ E-mail: tejoum@ciam.ru
}

Received December 28, 2004; revised May 15, 2005

\begin{abstract}
A Fedorenko Finite Superelements Method (FSEM) for 3D linear elasticity problems is considered. We present special weak statement for traces of original problem solution which natural Bubnov-Galerkin approximation leads to the FSEM. Some applications of the FSEM for the problems of mechanics of composites are considered and results of numerical experiments are presented.
\end{abstract}

Key words: Finite elements method, finite superelements method, elasticity problems

\section{Introduction}

In this work we discuss some applications of a Fedorenko Finite Superelements Method (FSEM) for 3D linear elasticity problems. We consider a simple case of isotropic inhomogeneous linear model for continuous media.

FSEM has been proposed in works of Fedorenko (see [8]) as a numerical approach for solving problems which solutions have local sharp singularities concentrated in relatively small subdomains of original computational domain.

In this work we generalize the approach which was used previously in $[11$, $12,13,14]$ for linear elasticity problems. All works share the same theoretical background for construction of FSEM approximations. As examples of applied problems we consider some problems of mechanics of a composite media. Let us note that simulation of displacement field of an composite media were

\footnotetext{
${ }^{1}$ The work was done under partial financial support of Russian Fund for Basic Research (project 03-01-00461).
} 
performed in original works of Fedorenko ([17]). Nevertheless, only qualitative analysis of the numerical method and results of simulation were presented. In this work we present some results on quantitative comparison between numerical results which were obtained and analytical and experimental models of behaviour of composite media.

We would like to thank Prof. Kuvyrkin for his suggestions and attention to this work.

\section{Problem Statement}

Our goal is to find displacement, stress and strain field in a bounded domain $\Omega \in \mathbb{R}^{3}$ with some displacements and forces applied at boundary $\partial \Omega$ of $\Omega$. We use the following model for a linear elastic media:

$$
\begin{aligned}
& \sigma_{i j, j}+f_{i}=0, \\
& \sigma_{i j}=2 \mu \epsilon_{i j}+\lambda \epsilon_{k k} \delta_{i j}, \\
& \epsilon_{i j}(u)=1 / 2\left(u_{i, j}+u_{j, i}\right),
\end{aligned}
$$

where $\partial A / \partial x_{i} \equiv A_{, i}$ for some $A=A\left(x_{i}\right)$. Also we will use the following operator form of (2.1):

$$
\operatorname{div} \sigma+f=0 .
$$

Here $\sigma=\sigma_{i j}$ is an elastic stress tensor, $\epsilon=\epsilon_{i j}$ is a strain tensor, $f$ is some forcing, $\lambda=\lambda\left(x_{i}\right), \mu=\mu\left(x_{i}\right)$ are Lame coefficients, $I=\delta_{i j}$ is the identity tensor.

At the boundary $\Gamma=\partial \Omega$ the following boundary conditions are defined:

$$
\left.u\right|_{\Gamma_{g}}=u_{g} \text { on } \Gamma_{g},\left.\quad \gamma_{\nu} \sigma\right|_{\Gamma_{n}}=f_{g} \text { on } \Gamma_{n} ; \quad \Gamma=\Gamma_{g} \cup \Gamma_{n},
$$

where $\nu=\left\{\nu_{j}\right\}$ is outwards normal to $\Gamma$.

\section{Classical Weak Statement}

Further we will use the following Green's formula ([18]):

$$
(\sigma, \epsilon(u))+(\operatorname{div} \sigma, u)=\left\langle\gamma_{\nu} \sigma, \gamma_{0} u\right\rangle,
$$

where

$$
(\xi, \eta)=\int_{\Omega} \xi \eta d \Omega, \quad\langle\xi, \eta\rangle=\int_{\partial \Omega} \xi \eta d \Gamma .
$$

Here $\xi \eta$ is a dot product in some Euclidean space $X$. In our case either $X=\mathbb{R}^{3}$ and then

$$
\xi \eta=\xi_{i} \eta_{i},
$$

or $X=E_{3}$ is a space of symmetric tensors of rank 3 , and then

$$
\xi \eta=\xi_{i j} \eta_{i j}
$$


$\gamma_{\nu} \sigma=\left.\sigma \nu\right|_{\partial \Omega}$ are normal stresses at $\partial \Omega, \gamma_{0} u=\left.u\right|_{\partial \Omega}$ is displacement field at the boundary.

Formula (3.1) is valid for any sufficiently smooth displacement field $u$, linear dependency $\epsilon(u)$ of the form (2.2) and arbitrary dependency $\sigma(\epsilon)$.

Let $V$ be a space of sufficiently smooth vector fields in $\Omega$, and $V_{0, g}$ consists of such elements in $V$ which vanish at $\Gamma_{g}$.

Using Green's formula the following weak statement can be obtained: find displacement field $u \in V$, such that

$$
(\sigma(u), \epsilon(v))=(f, v)+\left\langle f_{g}, \gamma_{0} v\right\rangle_{\Gamma_{n}}, \quad \forall v \in V_{0, g},\left.\quad u\right|_{\Gamma_{g}}=u_{g} .
$$

\section{Weak Statement for Traces}

According to FSEM a computational domain should be decomposed into a number of subdomains, so called superelements (SE). Then all unknowns are considered only at SE boundaries. To eliminate any unknowns defined inside SE Poincaré-Steklov (P.-S.) operator is used.

Poincaré-Steklov operator is defined as follows:

$$
P \varphi=\left.\gamma_{\nu} \sigma(G \varphi)\right|_{\partial \Omega}
$$

where $G$ is the Green operator. Green's operator maps an arbitrary field $\varphi$ defined at the boundary of domain to a field $u$ defined in whole domain, here $u$ is a solution of problem (3.2) with $\varphi$ as boundary condition and $f=0$.

P.-S. operator maps some function defined on SE boundary to another function defined on the same boundary. For any $\varphi, P \varphi$ is a normal stress at the SE boundary caused by boundary displacement field $\varphi$, i.e. P.-S. operator maps Dirichlet BCs into the equivalent Neumann ones.

Given Green's formula and definition of P.-S. operator we obtain the equality

$$
(\sigma(G \varphi), \epsilon(v))=\left\langle P \varphi, \gamma_{0} v\right\rangle
$$

which is valid for arbitrary sufficiently smooth field $v$. This formula can be used for numerical computation of values of P.-S. operators in an implementation of this method.

Now we will construct a weak statement of the problem. The BubnovGalerkin approximation of this problem leads to FSEM. Just for simplicity we consider only homogeneous problem, i.e. the case $f=0$. Generalization to the case $f \neq 0$ is straightforward.

Let $\Omega_{k}$ be superelements, $\Omega=\cup \Omega_{k}, \Gamma_{k}=\partial \Omega_{k}$ be a boundary of $\Omega_{k}$ and $\Gamma=\partial \Omega$ be a boundary of $\Omega$. Let $P_{k}, G_{k}, \gamma_{0, k}$ be $P, G, \gamma_{0}$ operators for some superelements $\Omega_{k}$. For arbitrary $v$ defined in $\Omega$ we use notation $v_{k}=\left.v\right|_{\Omega_{k}}$. For any sufficiently smooth field $w \in V$ denote by $\varphi_{k}$ a restriction of this field on $\Gamma_{k}$, i.e. $\varphi_{k}=\left.w\right|_{\Gamma_{k}}$.

In every $\mathrm{SE} \Omega_{k}$ let us consider the field $\tilde{w}$, which is defined as 


$$
\left.\tilde{w}\right|_{\Omega_{k}}=G_{k} \varphi_{k} .
$$

Suppose that $\tilde{w}$ is sufficiently smooth, i.e. $\tilde{w} \in V$. By definition $\tilde{w}$ is smooth at an interface boundary between two arbitrary SEs.

Moreover, $\tilde{w}$ is an exact solution of the problem under consideration in every separate SE $\Omega_{k}$. Nevertheless $\tilde{w}$ is not a solution of this problem in whole domain $\Omega$. Field $\tilde{w}$ will be a solution if it satisfies equation (3.2).

Substituting $\tilde{w}$ in (3.2) and taking into account that $\left.\tilde{w}\right|_{k}=G_{k} \varphi_{k}$ we obtain

$$
\begin{aligned}
(\sigma(\tilde{w}), \epsilon(v))) & =\sum_{k}\left(\sigma\left(\tilde{w}_{k}\right), \epsilon\left(v_{k}\right)\right)_{k} \\
& =\sum_{k}\left\langle\gamma_{\nu, k} \sigma\left(\tilde{w}_{k}\right), \gamma_{0, k} v_{k}\right\rangle_{k}-\sum_{k}\left(\operatorname{div} \sigma\left(\tilde{w}_{k}\right), v_{k}\right)_{k} \\
& =\sum_{k}\left\langle P_{k} \varphi_{k}, \gamma_{0, k} v_{k}\right\rangle_{k}-\sum_{k}\left(\operatorname{div} \sigma\left(G_{k} \varphi_{k}\right), v_{k}\right)_{k} \\
& =\sum_{k}\left\langle P_{k} \varphi_{k}, \gamma_{0, k} v_{k}\right\rangle_{k}=\left\langle f_{g}, \gamma_{0} v\right\rangle_{\Gamma_{n}}, \quad \forall v \in V_{0, g} .
\end{aligned}
$$

In order to be a solution the field $\tilde{w}$ should satisfy the following equation

$$
\sum_{k}\left\langle P_{k} \varphi_{k}, \gamma_{0, k} v_{k}\right\rangle_{k}=\left\langle f_{g}, \gamma_{0} v\right\rangle_{\Gamma_{n}} \quad \forall v \in V_{0, g}
$$

Taking into account that the equation above consists of quantities defined only at SE boundaries we can write problem in the following form: find $\varphi \in V_{\Gamma}$, such that

$$
\sum_{k}\left\langle P_{k} \varphi_{k}, \psi_{k}\right\rangle_{k}=\left\langle f_{g}, \psi\right\rangle_{\Gamma_{n}} \quad \forall \psi \in V_{\Gamma, 0} ;\left.\quad \varphi\right|_{\Gamma_{g}}=u_{g}
$$

where

$$
\begin{array}{cc}
V_{\Gamma}=\left\{\varphi=\left\{\varphi_{k}\right\}:\right. & \left.\varphi_{k}=\left.w\right|_{\Gamma_{k}}, \text { for some } w \in V\right\}, \\
V_{\Gamma, 0}=\left\{\varphi=\left\{\varphi_{k}\right\}:\right. & \left.\varphi_{k}=\left.w\right|_{\Gamma_{k}}, \text { for some } w \in V_{0, g}\right\} .
\end{array}
$$

Here $\varphi \in V_{\Gamma}$ is a set of functions $\varphi_{k}$, every of which is defined at $\Gamma_{k}$. If $V_{\Gamma, k}$ is a space of functions defined at $\Gamma_{k}$ and which are traces of function from $V_{k}$, then $\varphi \in \prod V_{k}, V_{\Gamma} \subset \prod V_{k}$. Also we have by definition that

$$
\left.\varphi_{k}\right|_{\Gamma_{k l}}=\left.\varphi_{l}\right|_{\Gamma_{k l}}, \quad \Gamma_{k l}=\Gamma_{k} \cap \Gamma_{l} .
$$

Equation (4.2) is a weak condition of a static equilibrium of superelements. Rewriting (4.2) and using new notation we obtain the following problem to be solved for $\varphi$ :

$$
\varphi \in V_{\Gamma}: \quad b(\varphi, \psi)=F(\psi), \quad \forall \psi \in V_{\Gamma, 0} ;\left.\quad \varphi\right|_{\Gamma_{g}}=u_{g},
$$

where $b(\cdot, \cdot)$ and $F(\cdot)$ are given by

$$
b(\varphi, \psi)=\sum_{k}\left\langle P_{k} \varphi_{k}, \psi_{k}\right\rangle_{k}, \quad F(\psi)=\left\langle f_{g}, \psi\right\rangle_{\Gamma_{n}} \quad \varphi, \psi \in V_{\Gamma} .
$$


Thus we have reduce original problem (3.2) in the whole domain $\Omega$ to the problem (4.3) which is defined only at SE boundaries. Problem (4.3) has a form of a variational equation. Theory of abstract variational equation is well developed as a theoretical background of finite element approximations. This theory can be used to obtain error estimates, but this is not a purpose of this work.

\section{Finite Dimensional Problem}

Finite superelement method can be considered as a projection method for solving problem (4.3). Further we consider only Bubnov-Galerkin approximations, i.e. the case when spaces of basis and trial functions are the same. More general PetrovGalerkin approximations can be considered in the same way.

In order to construct the approximation of the variational problem some finite dimensional space $V_{\Gamma, h} \in V_{\Gamma, h}$ has to be defined. Let $\left\{\varphi_{h, i}\right\}$ be a set of basis functions, such that

$$
V_{\Gamma, h}=\operatorname{span} \varphi_{h, i} .
$$

An approximate solution has the following form

$$
\varphi_{h}=\sum_{i} a_{i} \varphi_{h, i}
$$

and it is a solution of the finite-dimensional problem

$$
\varphi_{h} \in V_{\Gamma, h}: \quad b\left(\varphi_{h}, \psi_{h}\right)=0, \quad \forall \psi_{h} \in V_{\Gamma, 0, h} ;\left.\quad \varphi_{h}\right|_{\Gamma, g}=u_{g} .
$$

This problem can be considered as a system of linear algebraic equations for $a_{i}$.

Solution of this problem can be performed in two steps. First we compute $P \varphi_{h, i}$ for every $i$ in each SE $\Omega_{k}$. This can be done by solving problem (3.2) in every $\Omega_{k}$ and taking $\varphi_{h, i}$ as boundary conditions. Finite difference or finite element approximations can be used. All these problems can be solved independently so parallelization of FSEM is quite straightforward.

The second step is to compute SE stiffness matrices and assemble a full stiffness matrix. To compute stiffness matrices of SE the definition of P.-S. operators can be used but it is more convenient to use equation (4.1) instead. In this case we avoid numerical differentiation of Green's operator.

A solution of the variational discrete problem in the whole domain $\Omega$ then is given by

$$
u_{h}=\sum_{i} a_{i} G \varphi_{h, i} .
$$

Let us note that there is no need to compute functions $G \varphi_{h, i}$ at this step. It has been already done during procedure of computing P.-S. operators at the step one.

In general FSEM computational procedure follows FEM one. The only difference is that one have to precompute SE basis function before applying stiffness matrix assembling procedure.

In this work we use 2D bilinear approximations at the SE boundaries. All SEs are brick-shaped and every face is considered as a cell of 2D boundary mesh. In general case one can use standard 2D approaches and finite elements to construct the mesh of boundary finite elements. 


\section{Numerical Simulation}

In this section we apply computational approach described above for simulation of displacement, stress and strain fields in a composite media reinforced with short fibers. We are interested to compute averaged elastic properties of composite as well as to investigate its micromechanical behaviour.

Many approaches are known for calculation of effective properties of composites with given structure and elastic properties of its components. We can mention asymptotic homogenization approach ([3]), numerous "fusion" ([4, 6, 7, 20]) and micromechanical models $([1,2,5,9,16])$ which are widely used in engineering practice.

We use theoretical models with known effective composite properties from [4, 6, $7,20]$ for validation of our computational results.

The method of cells can be used for simulation of the micromechanical behaviour of a composite (see [10] and references therein). This approach is based on the analysis of a repeating cell in a composite. In order to obtain stress/strain field or effective properties of a media some model problems have to be solved in one cell of composite.

FSEM let us to perform direct numerical simulation of a composite media with a large number of fibers without applying any homogenization procedure. As a result of simulation we obtain detailed displacement/stress/strain field in a media as well as media effective properties.

Let us point out that application of a conventional FEM method for this problem is quite difficult because it leads to a computational problem of very large dimension. To resolve all singularities of a solution one has to use very fine computational mesh with about $\sim 10^{5}$ equations per one cell of a composite. If we want to obtain a good quality solution in a computational domain with several inclusions, then we have to solve a computational problem, which consists of $\sim 10^{5}-10^{7}$ equations and such a problem is unacceptably large.

Following FSEM procedure we decompose computational domain into a number of subdomains - superelements. Every such SE describes one cell of a composite and contains one inclusion (fiber). In general case all SEs (and corresponding fiber) are different i.e. they can have different geometry and elastic properties. In this work all SEs are cubes, fibers have spherical or brick-shaped geometry. Elastic properties of matrix and fiber don't change from one SE to other. Every inclusion is contained inside one SE. In this case solution of a problem is smooth at the interfaces between different SE, so simple bilinear interpolation at SEs boundaries can be used. To resolve all singularities of a solution in a particular SE (i.e. at the matrix-fiber interface) a fine adaptive FE mesh is introduced in every SE. These FE meshes are defined independently in every SE.

In the rest of this paper we consider some numerical results concerning determination of effective media properties and direct simulation of behaviour of some particular composite medias. Obtained results are compared with some theoretical and experimental estimates.

To determine effective elastic properties we use the following procedure. At the boundary of the computational domain we define boundary conditions which, in a 
case of homogeneous and isotropic media, leads to uniaxis stress/strain state. Then effective properties can be calculated via comparison of a simulated solution for composite media and explicit solution of this problem for homogeneous and isotropic media. More detailed explanation is presented in [15].

In all simulations the computational domain contains from 125 to 3375 superelements (from $5 \times 5 \times 5$ to $15 \times 15 \times 15$ superelement cells).

The first case we consider is the composite media which consists of a number of SEs of the same type, i.e. all SE are cubes, fibers are either cubes or spheres of the same size, geometry, orientation and elastic properties. The only parameter we change is fiber volume ratio.

On Fig. $1 a$ the experimental and simulated values of Young modulii are presented as well as some theoretical estimates. Experimental values are denoted as a diamond marker, simulated ones as circles. We use experimental values from [19]. Elastic modulii are the following

$$
E_{m}=1.0, \mu_{m}=0.29, \quad E_{f}=3.41, \quad \mu_{f}=0.22 .
$$

It can be seen that simulated values are in a good agreement with the experimental ones. On this figure some analytical estimates are presented too (Foight-Reissner, Hashin-Shtricman estimates and estimates obtained by Kuvyrkin, ([4, 6, 7, 20]). In this simulation all fibers are cubes of the same size.

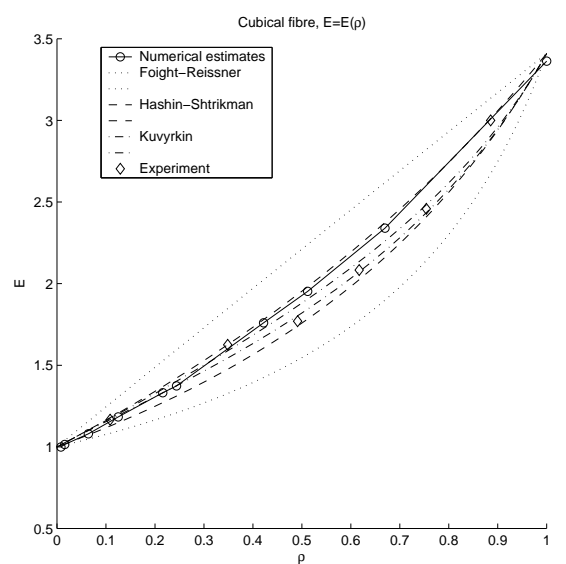

a)

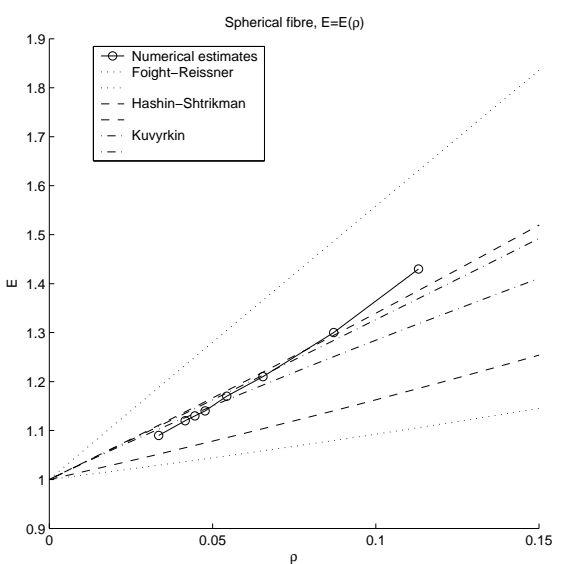

b)

Figure 1. Experimental and simulated Young modulii: a) all SE are cubes, b) composite with $A l$ matrix and spherical $S i C$ fibers.

On Fig. $1 b$ the same dependency is presented for the case of a composite with $A l$ matrix and spherical $\mathrm{SiC}$ fibers. Elastic properties of matrix and fiber are given as

$$
E_{m}=1, \mu_{m}=0.33, E_{f}=6.5, \mu_{f}=0.33 .
$$

On Fig. $2 a$ plots for the same case as on Fig. $1 b$ are presented, but fibers are cubes. In this case comparison with the experimental estimates wasn't performed. 


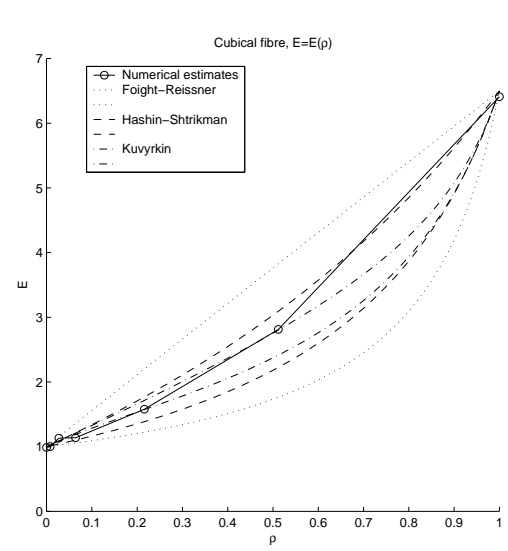

a)

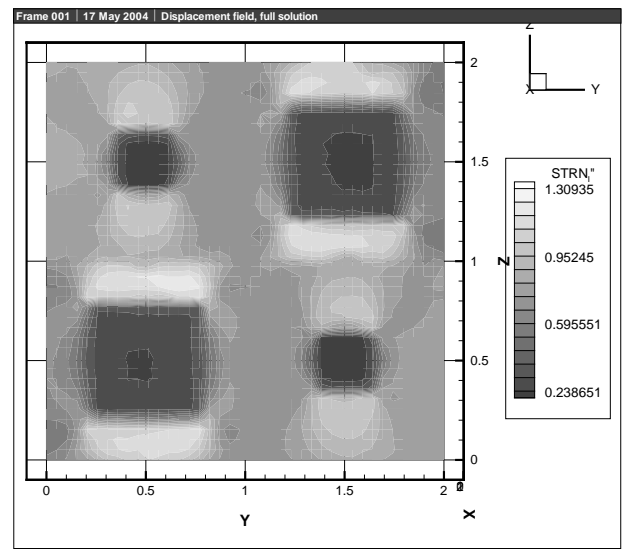

b)

Figure 2. a) Simulated values of Young modulii, cubic fibers $(A l-S i C)$, b) strain field at $x=0.5$.

Table 1. Theoretical estimates, $\rho=0.243$.

\begin{tabular}{cccc}
\hline & Foight-Reissner & Hashin-Shtricman & Kuvyrkin \\
\hline$E_{\min }$ & 1.2588 & 1.4447 & 1.6393 \\
$E_{\max }$ & 2.3494 & 1.8751 & 1.8074 \\
\hline$\mu_{\min }$ & 0.2819 & 0.2915 & 0.2994 \\
$\mu_{\max }$ & 0.3262 & 0.3120 & 0.3108 \\
\hline
\end{tabular}

The second case is composite media which consists of a number of SEs of different type, i.e. composite media reinforced with a brick-shaped fibers which have different orientation in space. The lengths of the fiber's edges are $h_{1}, h_{2}, h_{3}, h_{i} \| O x_{i}$ and $h_{1}=h_{2}=3 h_{3}$. Fiber can be oriented along one of the coordinate axis $O x_{i}$. In this case we have SE of three types which are corresponded to three variants of orientation of a fiber. Computational domain was randomly filled with these SEs. Fiber volume ratio in this simulation is $\rho=0.243$. Elastic properties of matrix and fiber are $E_{m}=1.0, \mu_{m}=0.33, E_{f}=6.5, \mu_{f}=0.25$. Simulated values of effective elastic modulii in this case are the following:

$$
E=1.8870, \quad \mu=0.2662,
$$

and for cubic fibers (and the same volume ratio)

$$
E_{c}=1.6832, \quad \mu_{c}=0.2796 .
$$

It is interesting to compare this simulated values with theoretical estimates mentioned above (Tab. 1). It can be seen that in the case of cubic fibers the simulated values are in good agreement with the theoretical boundaries. In this case the simulation is close to the assumptions under which theoretical estimates were obtained (local isotropy and homogeneity of a composite media). 
Differences of the simulated values in (6.1) and (6.2) show the influence of microlevel anisotropy of a media on effective elastic properties.

We obtained the following values of the Young modulii in the case when all fibers are oriented along one direction: $E=1.5408, \mu=0.2424$ (all fibers are oriented along $O z$ ), $E=2.0693, \mu=0.2934$ (all fibers are oriented along $O x$ or $O y$ ).

In the simulations described above the computational domain was randomly filled with SEs of different types so only one variant of random arrangement was used. Now we present some results which show how simulated values of effective properties depend on a way SE are arranged into a whole computational domain. We consider computational domains with $5 \times 5 \times 5,10 \times 10 \times 10,15 \times 15 \times 15$ superelements. For every domain 10 random arrangements of SE were generated. Results are presented in table Tab. 2. For every domain a mean value of Young modulii, the Poisson coefficient and the maximum difference of particular and mean value for all simulation for certain domain are shown.

Table 2. Mean values of elastic coefficients.

\begin{tabular}{rcccc}
\hline & $E$ & $\Delta E$ & $\mu$ & $\Delta \mu$ \\
\hline $5 \times 5 \times 5$ & 1.8946 & 0.031583 & 0.272 & 0.02852 \\
$10 \times 10 \times 10$ & 1.8899 & 0.0093559 & 0.27736 & 0.018883 \\
$15 \times 15 \times 15$ & 1.8883 & 0.0064842 & 0.27262 & 0.0099899 \\
\hline
\end{tabular}

One can see that difference between particular and mean values decreases very fast as a number of SE increases.

Finally we present a typical solution of the given applied problem. On Fig. $2 b$ a distribution of intensity of strain tensor in a computational domain is shown. This picture corresponds to a slice of the computational domain with a plain $x=0.5$. Computational domain consists of eight SEs $(2 \times 2 \times 2)$. Fibers are cubes of a different size.

In all simulations $\mathrm{SE}$ basis functions were computed with conventional FE approach. We have used simple linear finite elements on a thetraedral mesh. To resolve solution on the matrix-fiber interfaces adaptive meshes were used. The number of nodes in such mesh was about $\sim 10^{5}$.

\section{References}

[1] J. Aboudi. A continuum theory for fiber-reinforced elastic-viscoplastic composites. Int. J. Engng. Sci., 20(5), 605 - 621, 1982.

[2] J. Aboudi. Micromechanical analysis of composites by the method of cells. Appl. Mech. Rev., 42(7), 193 - 221, 1989.

[3] N.S. Bakhvalov and G.P. Panasenko. Homogenization of processes in periodic media. M.: Nauka, 1984. (in Russian)

[4] N.G. Burago, M.P. Galanin and G.N. Kuvyrkin. Basic variational principles of mechanics of continuous medias. M.: MSTU n.a. Bauman, 2004. (in Russian) 
[5] R. Christensen. Introduction into mechanics of composites. M.: Mir, 1982. (in Russian)

[6] A.V. Efimenko and G.N. Kuvyrkin. New estimates of the effective elastic modulii of two-component composites. Izv. RAN. Mekhanika Tverdogo Tela, 29(1), 18 - 26, 1994. (in Russian)

[7] A.V. Efimenko, V.S. Zarubin and G.N. Kuvyrkin. On bilateral estimates of thermomechanical and thermophysical properties of nonhomogenious materials. Dinamika, prochnost' i iznosostoikost' mashin. International journal on electronic medias, 2, 3 - 7, 1996. (in Russian)

[8] R.P. Fedorenko. Introduction into computational physics. M.: MFTI, 1994. (in Russian)

[9] W.J. Fleming and A.L. Dowson. Prediction of the fatigue life of an aluminum metal matrix composite using the theory of cells. Science and Engineering of Composite Materials, 8(4), 181 - 189, 1999.

[10] W.J. Fleming and J.M. Temis. Numerical simulation of cyclic plasticity and damage of an aluminum metal matrix composite with particulate $\mathrm{SiC}$ inclusions. Int. Journal of Fatigue, 24, 1079 - 1088, 2002.

[11] M. Galanin and E. Savenkov. On relations of finite superelements and projection methods. Preprint of KIAM of RAS, 67, 2001. (in Russian)

[12] M. Galanin and E. Savenkov. Fedorenko finite superelement method as special Galerkin approximation. Mathematical Modelling and Analysis, 7(1), 41 - 50, 2002.

[13] M. Galanin and E. Savenkov. Substantiation of the finite superelements method. J. of Comp. Math. and Math. Phys., 43(5), 711 - 727, 2003. (in Russian)

[14] M. Galanin and E. Savenkov. Finite superelements method for velocity skin-layer problem. Preprint of KIAM of RAS, 11, 2004. (in Russian)

[15] M. Galanin, E. Savenkov and J. Temis. Fedorenko finite superelements method for elasticity problems. Preprint of KIAM of RAS, 38, 2004. (in Russian)

[16] V.R. Skvortcov. Foundations of mechanics of composites. SPb.: SPbGMTU, 1995. (in Russian)

[17] L.G. Strakhovskaja and R.P. Fedorenko. The calculation of the stress state of 3D composite body by Finite Superlements Method. Preprint of KIAM of RAS, 97, 1994. (in Russian)

[18] R. Temam. Mathematical problems of plasticity theory. M.: Nauka, 1991. (in Russian)

[19] V.S. Zarubin. Applied problems of thermostrength of constructions. M.: Nauka, 1977. (in Russian)

[20] V.S. Zarubin and G.N. Kuvyrkin. Prognoses of thermophysical and thermoelastic properties of composites. Vestnik MGTU. Ser. Mashinostroenie, 2, 78 - 83, 1994. (in Russian)

\section{Baigtinių superelementų metodas elastiškumo uždaviniams}

M. Galanin, E. Savenkov, Yu. Temis

Straipsnyje nagrinėjamas Fedorenkos baigtinių superelementų metodas trimačiams tiesiniams elastiškumo uždaviniams. Pasi-ulytas specialus silpnasis sprendinio jedsako formulavimas, kuris iprastą Bubnovo-Galerkino aproksimaciją susieja su baigtinių superelementų metodu. Nagrinejjami kai kurie baigtinių superelementų metodo taikymai kompozitų mechanikos uždaviniams. Pateikti skaitinio eksperimento rezultatai. 